This is a pre-print of a contribution published in "Robot 2019: Fourth Iberian Robotics Conference. Advances in Intelligent Systems and Computing, Vol 1093. Silva M., Luís Lima J., Reis L., Sanfeliu A., Tardioli D. (eds)" published by Springer, Cham. The final authenticated version is avalaible online at: https:/doi.org/10.1007/978-3-030-36150-1_36

\title{
Grasping Angle Estimation of Human Forearm with Underactuated Grippers Using Proprioceptive Feedback
}

\author{
Francisco Pastor, Juan M. Gandarias, Alfonso J. García-Cerezo, \\ Antonio J. Muñoz-Ramírez, and Jesús M. Gómez-de-Gabriel \\ Telerobotic and Interactive Systems Laboratory, System Engineering and Automation \\ Department, University of Málaga, Málaga 29071, Spain, \\ \{fpastor, jmgandarias, ajgarcia, aj, jesus.gomez\}@uma.es, \\ WWW home page: www.taislab.uma.es
}

\begin{abstract}
In this paper, a method for the estimation of the angle of grasping of a human forearm, when grasped by a robot with an underactuated gripper, using proprioceptive information only, is presented. Knowing the angle around the forearm's axis (i.e. roll angle) is key for the safe manipulation of the human limb and biomedical sensor placement among others. The adaptive gripper has two independent underactuated fingers with two phalanges and a single actuator each. The final joint position of the gripper provides information related to the shape of the grasped object without the need for external contact or force sensors. Regression methods to estimate the roll angle of the grasping have been trained with forearm grasping information from different humans at each angular position. The results show that it is possible to accurately estimate the rolling angle of the human arm, for trained and unknown people.
\end{abstract}

Keywords: pHRI, underactuated gripper, propioceptive sensors, regression, haptic perception.

\section{Introduction}

Triage and initial care are crucial for victims in disaster scenarios [10. These tasks frequently need safe human limb manipulation. Although visual methods for the estimation of the location of human limbs provide the coordinates of the human joints [7, the angle around the forearm axis (roll angle) is not included. Moreover, the roll of the forearm may change during the grasping process.

Despite the fact that the applications where robots are able to manipulate people are very interesting for robotics researchers, there are few studies that consider direct human-robot physical contacts. Most research studies that are related to physical Human-Robot Interaction (pHRI) consist of control techniques of teleoperated systems [5], exoskeletons [15], prosthetic parts or rehabilitation robots [19]. 


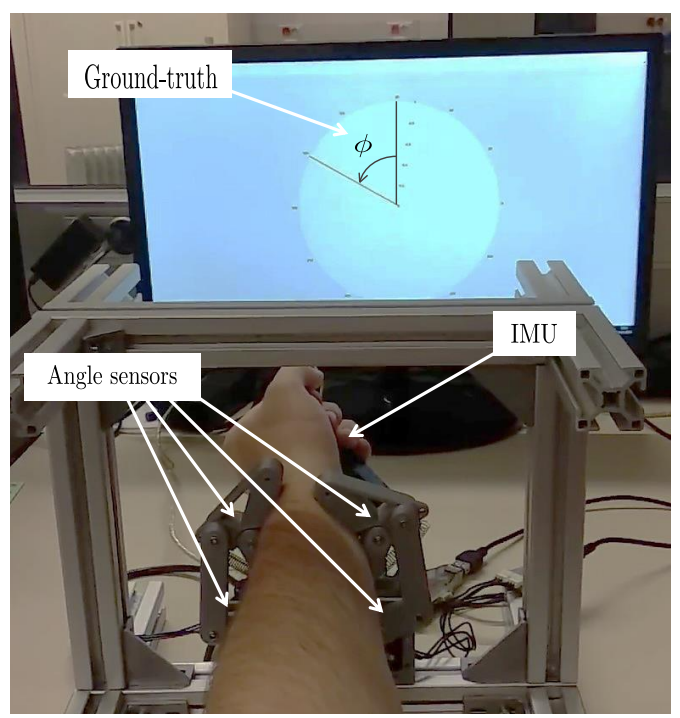

Fig. 1. Adaptive gripper for grasping angle estimation of human forearm. Proprioceptive sensors (angle sensors) are used to estimate the roll-angle, while an IMU is used as ground-truth to train the estiamtion methods.

Regarding these few pHRI applications, a robot that cleanses human limbs of disabled people is presented in [13] and a robot that manipulates human limbs with a non-prenssil actuator and an impedance Model Predictive Control is presented in [8]. More recent studies have considered the application of artificially intelligent techniques for robotically assisted dressing but without robot-human contact 9 .

One aspect that has to be addressed in pHRI refers to robotics grippers. Although multiple ad-hoc end-effectors have been considered in previous pHRI works [21, it is still necessary to develop grippers or hands that allow the robot to carry out autonomous and safe grasps [1, with enough robustness and softness to manipulate human limbs. Some studies about pHRI grippers are based on the use of Variable Stiffness Actuators (VSA) [17, and the integration of tactile sensors and deep learning methods in robotic grippers to distinguish contacts with humans and inert objects is presented in [12].

The use of adaptive or flexible grippers enhance the in-hand manipulation by reducing the maximum pressure applied over the grasped objects [11. However, the precision of this kind of mechanism is lower than in rigid grippers. Other existing solutions are based on underactuated rigid hands that adapt their shape over the contact surface. The underactuated and fully rigid PaCome gripper [2, originally thought for industrial applications, could also be used for pHRI applications. In [16, OpenHand is presented. It is an hybrid rigid-soft and open hardware gripper made of 3D printed polylactic acid (PLA) and polyurethane rubber. In a recent study of the same authors, proprioceptive and tactile in- 
formation are combined to classify grasps with a hybrid underactuated gripper [18.

In this paper, the problem of human forearm manipulation is addressed. To face this task, a gripper with two independent underactuated fingers has been designed and built using additive manufacturing technology. Each finger has two phalanxes, a single motor and an angle sensor placed in the underactuated joint. The main contribution of this study is to solely use proprioceptive information given by angle sensors integrated in the gripper to grasp the distal forearm and estimate the roll angle using machine learning approaches based on non-linear regression methods. According to this study, a robot with an underactuated gripper could grasp a distal forearm and estimate the grasping roll angle using haptic feedback only, which could open the door to new research lines into safety human manipulation.

This paper is structured as follows: In section 2 the designed prototype of the underactuated gripper and its validation is presented. In section 3 the new method developed for the estimation of the forearm roll angle, based on machine learning techniques is then described, and the conclusions are shown in section 4.

\section{Adaptive Gripper}

There are two main approaches for the implementation of the driving mechanism: tendons and rigid linkages [4. In this application, the use of tendons (e.g. Yale OpenHand Model T42), as in [16] has been discarded, because the tendons are located in the internal side of the fingers, so the contact surfaces of the fingers tend to pinch the skin of the forearm. Rigid linkages has been used, because they are located on the back of the finger, leaving a cleaner contact area.

\section{$2.1 \quad$ Kinematics}

A gripper with two independent underactuated fingers with two phalanx and a single actuator has been designed with the kinematics described in Figure 2 . The parameter values, summarised in Table 1, have been designed to adapt to the shape and size of a human upper-forearm with a perimeter between 15.3 and $18.8 \mathrm{~cm}$.

Table 1. Parameter values of the underactuated finger.

\begin{tabular}{|c|c|c|c|c|c|c|c|c|}
\hline Parameter & $\overline{\boldsymbol{O}_{1} \boldsymbol{O}_{\boldsymbol{a}}}[\mathrm{mm}]$ & $L_{0}, L_{1}, L_{2}$ & $\boldsymbol{2}[\mathrm{mm}] \boldsymbol{a}[\mathrm{mm}]$ & $\boldsymbol{b}[\mathrm{mm}]$ & $\boldsymbol{c}[\mathrm{mm}]$ & $\boldsymbol{d}[\mathrm{mm}]$ & $\psi{ }^{\circ}$ & $\mathrm{dth}[\mathrm{mm}]$ \\
\hline Value & {$[16,-20]$} & 40 & 25 & 60 & 20 & 8 & 90 & 15 \\
\hline
\end{tabular}

A mechanical limit makes the distal phalanx angle $\theta_{2}$ always positive. The actual position of the finger depends on the balance between external forces 


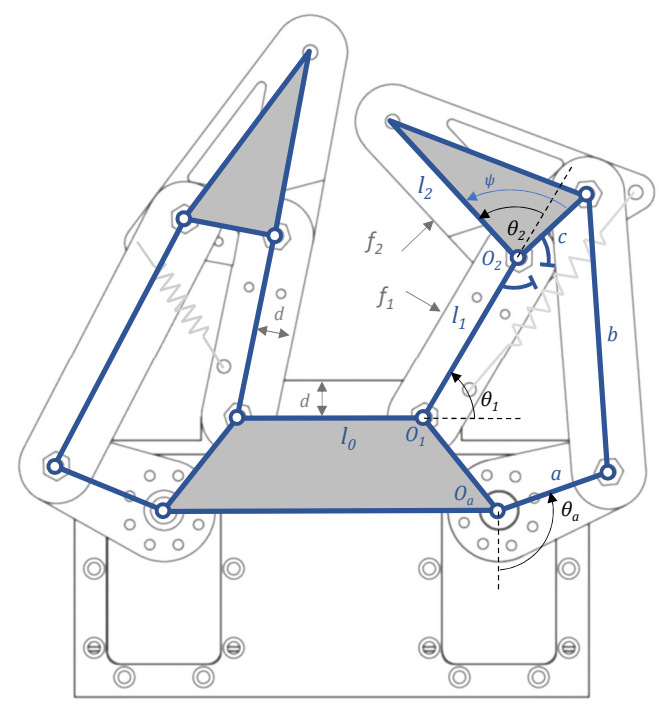

Fig. 2. Kinematics of the underactuated fingers. Each finger is independent, with two DOF's $\left(\theta_{1}, \theta_{2}\right)$ and a single actuator $\theta_{a}$. A mechanical stop makes $\theta_{2}>0$. The actual position of the finger depends on the external forces $f_{1}, f_{2}$ and the actuator torque. A spring ensures contact between the finger pads and external objects

$f_{1}, f_{2}$ and the actuator torque. The spring ensures contact between the finger pads and external objects and makes the finger stable when $f_{1}$ or $f_{2}$ are 0 . The extension springs are made with $0.6 \mathrm{~mm} \oslash$ steel wire and a stiffness of $164 \mathrm{~N} / \mathrm{m}$.

The prototype has been manufactured in PLA plastic in a Prusa MK2 3Dprinter, and its design has been made accessible in a public repository 1

\section{$2.2 \quad$ Proprioceptive sensing}

Analog angle sensors have been placed to measure the distal joint angles $\theta_{2}$. The actuators provide feedback on the servo position, so the full position of the adaptive fingers can be estimated. This way, proprioceptive joint sensors provide information about the final gripper position that is related to the shape and size of the grasped object, without the need of external contact or force sensors.

Miniature potentiometers from muRata (model SV01 $10 k \Omega$ linear) have been used successfully for the measurement of the distal joints of both fingers $\left(\theta_{2 l}, \theta_{2 r}\right)$. The analog signals are measured using a micro-controller with 10-bits ADC, $\left(0.26^{\circ}\right.$ resolution $)$ at a rate of $50 \mathrm{~Hz}$.

The Dynamixel $M X-28$ servos have a magnetic encoder with 12-bits $\left(0.088^{\circ}\right.$ resolution) at a rate of up-to $50 \mathrm{~Hz}$ with our current set-up. They provide feedback of the servo positions $\theta_{a l}$ and $\theta_{a r}$.

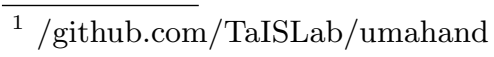




\subsection{Grasping forces}

The forces at the center of the phalanxes contact areas in an underactuated gripper depend also on the joint values. Moreover the spring stiffness [3] is also in the equation.

$$
f=J^{-T} T^{-T} t
$$

Where $f$ are the contact forces, $J^{-T}$ is the inverse of the transposed Jacobian matrix that relates the finger joint velocities to the speed of the contact points, and $\mathrm{T}$ is the transfer matrix, that relates the velocities of the actuators to the joint velocities. Both matrices depend on the joints and actuator positions [4. However, for a position of the gripper given $\left(\theta_{1}\right.$ and $\theta_{2}$ remains constant), the magnitude of the closing force of each finger $\left(f_{1}+f_{2}\right)$ is proportional to the actuator torque $\theta_{a}$. Closing forces have been experimentally measured for different actuator torques at the same grasping position. With a maximum stall torque for each of the Dynamixel MX-28 servos of $2.5 \mathrm{Nm}$, the effective closing forces for each finger range from $4.9 \mathrm{~N}(20 \%)$ to $27.4 \mathrm{~N}(100 \%)$.

\subsection{Getting ground-truth data}

In order to obtain the ground-truth angular measurement of the human forearm, a device that includes an accelerometer has been implemented. The device is held by the volunteers with their hand during the experiments. As the attitude of the robot gripper is known, the relative rolling angle of the human forearm with respect the gripper can be obtained and used as a reference data for training and performance evaluation of estimation methods.

\section{Forearm roll angle Estimation}

The proposed method is based on the differences in the final grasping positions of the finger joints when grasping a human forearm, thanks to the internal bone structure, as seen in Figure 3 . The human forearm is supported by Ulna and Radius, which specially at the upper section of the forearm (near the wrist) provide an elliptical shape. The roll angle is measured with respect to the pose in which Ulna and Radius are parallel to the gripper base.

\subsection{Measurements}

During the measurements, the volunteer subject holds a 3D printed handle which integrates the accelerometer, while the gripper closes repeatedly around their forearm. The subject has to rotate the forearm in each iteration, so that the accelerometer measures this new angle, which corresponds to the roll angle. The whole gripper has been mounted on a square frame to perform experiments in different positions. The actual roll angle is computed as the orientation difference between the gripper and the readings from the inertial sensor bar. This process 


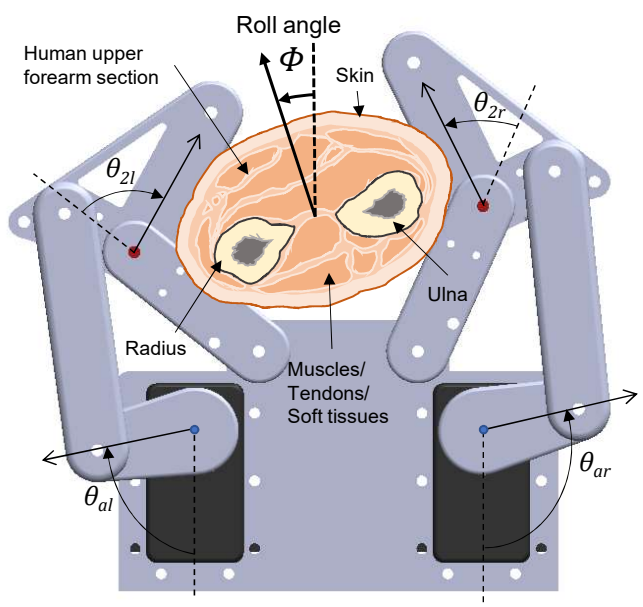

Fig. 3. Cross section of a human upper forearm grasped by the underactuated gripper, showing the variations in the passive $\left(\theta_{2 l}, \theta_{2 r}\right)$ and active $\left(\theta_{a l}, \theta_{a r}\right)$ DOF's, based on the roll grasping-angle $(\Phi)$.

is shown in Figure 4, where the arm angle and joint values are recorded for a sequence of six grasps.

Performing many different grasps at different angles, the relationship between the gripper joints and the roll can be obtained. In Figure 5 , the data of 36 grasps, measured at different roll angles, on the left arm of a volunteer with a perimeter of $17.9 \mathrm{~cm}$, is shown.

\subsection{Machine-learning regression methods}

Regression methods have been implemented due to To estimate the roll angle $(\phi)$, three machine learning approaches based on non-linear regression methods are used to obtain three models: Gaussian Process Regression (GPR) [20, Regression Tree (RT) [6] and Bagging Regression Tree (BRT) [14. These models receive the 4 -input angles $\left(\theta_{a r}, \theta_{a l}, \theta_{2 r}, \theta_{2 l}\right)$ and predict $\phi$.

All these models are trained in a large dataset. In the training process, features are composed by sets of $\left(\theta_{a r}, \theta_{a l}, \theta_{2 r}, \theta_{2 l}\right)$, while expected responses composed by $\phi$ are measured with the accelerometer for each set of features. A cross-validation has also been included during the training process to prevent overfitting.

The training and evaluation processes have been carried out using Matlab R2018b, the Statistics and Machine Learning Toolbox and the Regression Learner application. The code and datasets have been made available in the repository referred in section 2 ,

The training process have been carried out using the Parallel Computing Toolbox in a 4-core Intel i7-7700HQ CPU @ 2.80 GHz. 


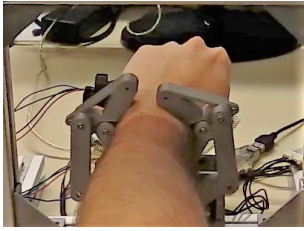

$5^{\circ}$

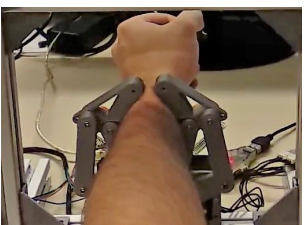

$90^{\circ}$

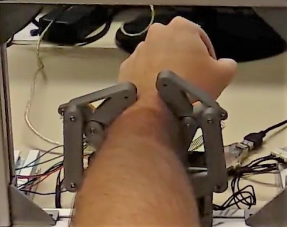

$35^{\circ}$

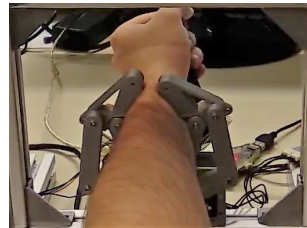

$120^{\circ}$

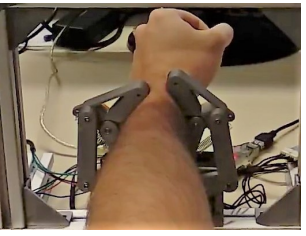

$55^{\circ}$

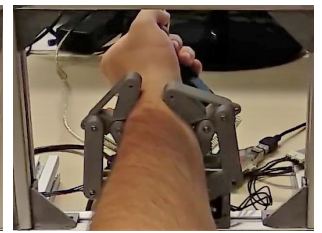

$140^{\circ}$

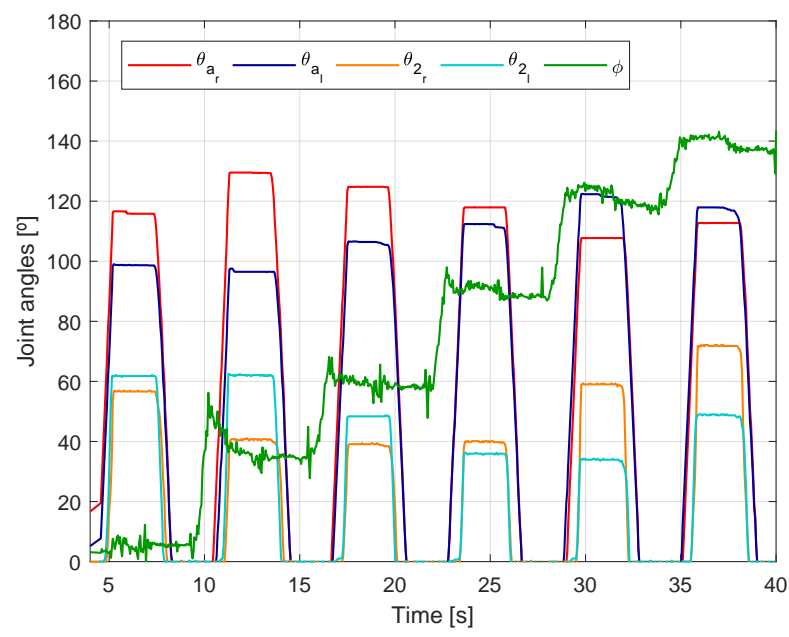

Fig. 4. Sequence of 6 grasps with different roll angles during the data collection process of one volunteer (top). Motors, joints and roll angle positions during the data collection process (bottom). Note that in this graph the open-and-close process is repeated each $6 \mathrm{~s}$. 


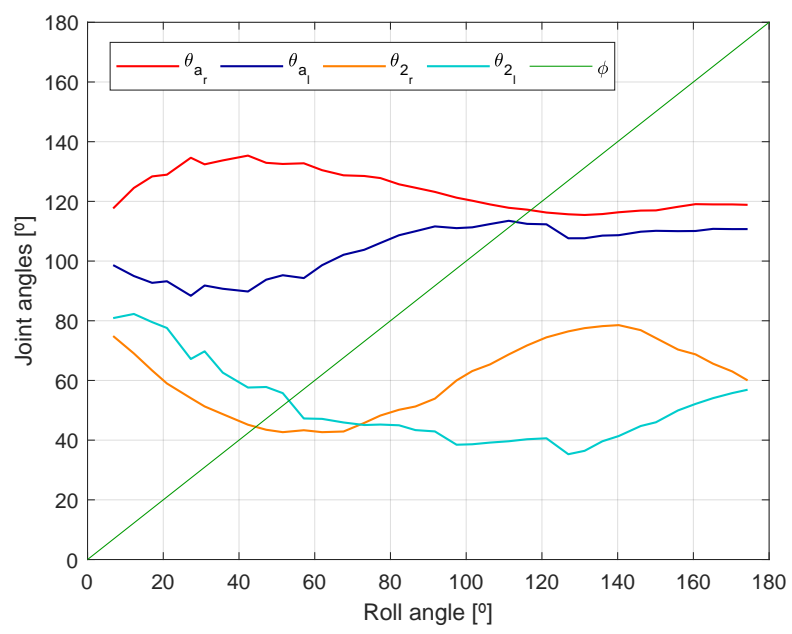

Fig. 5. Joint values for 36 grasps, measured at different angles, on the left arm of a volunteer with a perimeter of $17.9 \mathrm{~cm}$, against the roll angle.

\subsection{Data collection}

During this process, a User Interface developed in Matlab shows goals and current angles so that the subject could rotate their forearm until goal and actual angle match. In each step, the gripper opens and closes, so while the gripper is closed, $\left(\theta_{a r}, \theta_{a l}, \theta_{2 r}, \theta_{2 l}\right)$ and $(\phi)$ values are collected.

There are two types of data collection processes which have been performed: sequential and random. In the sequential process, the subject is asked to rotate their arm in steps of $5^{\circ}$. In the latter, the goal angle is set randomly.

Following this process, two data-sets have been collected: the first dataset contains information of a single subject, while the second dataset contains information of five subjects. To train and test the models, each dataset is split into training and test sets respectively. Therefore, training and test data are different, even if they have been collected from the same person.

The dataset collected from one subject contains an amount of 555 sets of $\left(\theta_{a r}, \theta_{a l}, \theta_{2 r}, \theta_{2 l}\right)$ and $(\phi)$, while the dataset collected from 5 subjects is composed by 2775 sets of data.

\subsection{Results}

Three experiments have been carried out to measure the performance of the regression models estimating the roll angle:

1. Single-known: Training and test sets contain data from one subject.

2. Multiple-known: Training and test sets contain data from four subjects.

3. Multiple-unknown: Training set contains data from four subjects and test set contains data from a fifth subject which is not used to train the models. 
In the single-known experiment, machine learning models have been trained with data from a single volunteer and have tested with different data from the same subject. The results of this experiment are shown in Fig. 6. This figure shows the good performance of the regression models, with an almost negligible error, and the best results obtained by GPR.

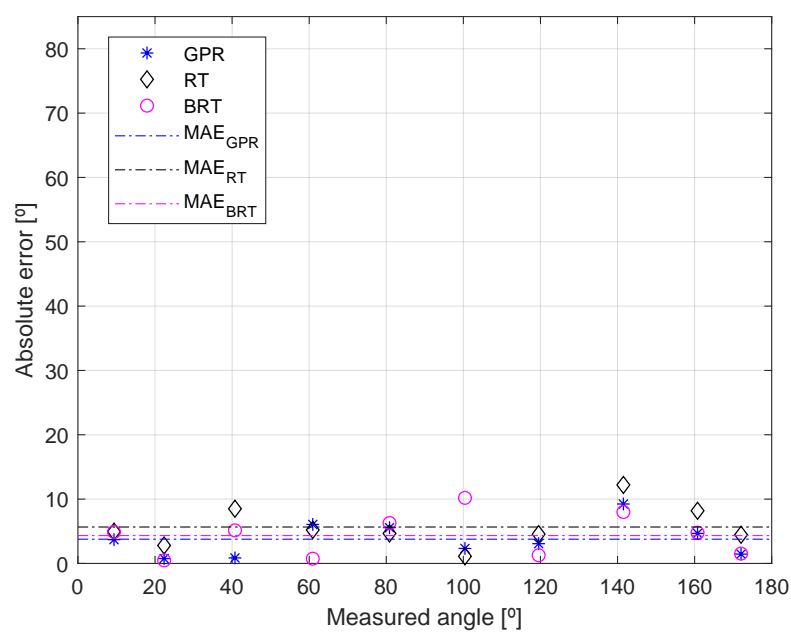

Fig. 6. Regression errors results of non-trained data of a known subject when models are trained in this subject dataset only.

In the multiple-known experiment, machine learning methods have been trained with data from 4 volunteers and have been tested with different data from one of these 4 subjects. In this case, predictions from RT and BRT include a pair of outliers, however the GPR model still presents a good performance as can be seen in Fig. 7.

In the multiple-unknown experiment, regression models have been trained with data from 4 subjects and tested with completely new data from a fifth volunteer that had not been used in the training process. Results of this experiment are presented in Fig 8 and show that RT and BRT are more robust that GPR because they generalize better, and the outliers predicted in the previous experiment vanish.

Results are summarized in Table 2. The Maximum Erorr (ME) and Mean Absolute Error (MAE) from each model in each experiment are represented in degrees. As is commented before, GPR obtains the best results when the subject is known, with a MAE of $3.77^{\circ}$ in the case of a single user and $8.68^{\circ}$ in the case of multiple subjects. However, for the third experiment, the best results are achieved by the RT model with a MAE of $9.86^{\circ}$ since this model generalizes better than the others. The maximum error shows the outliers in experiments 


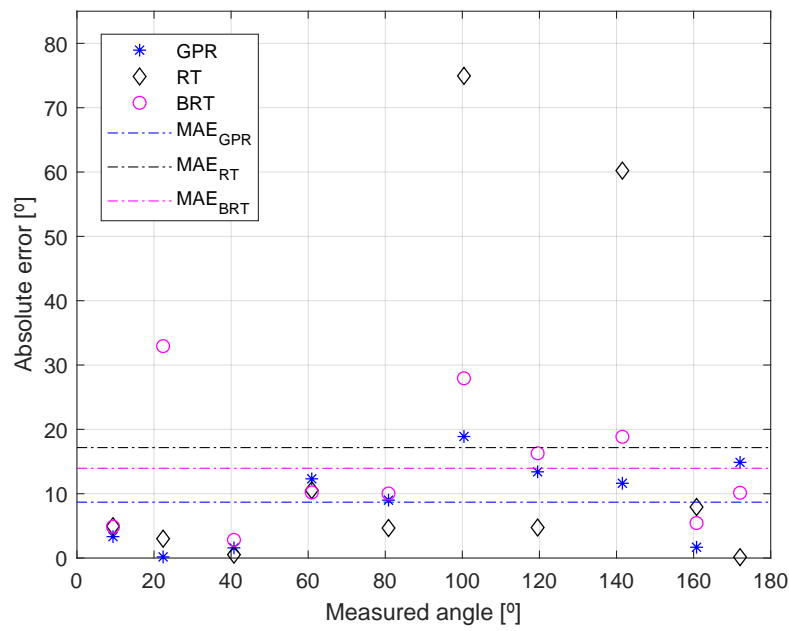

Fig. 7. Regression errors results of non-trained data of a known subject when models are trained in a dataset obtained from 4 volunteers.

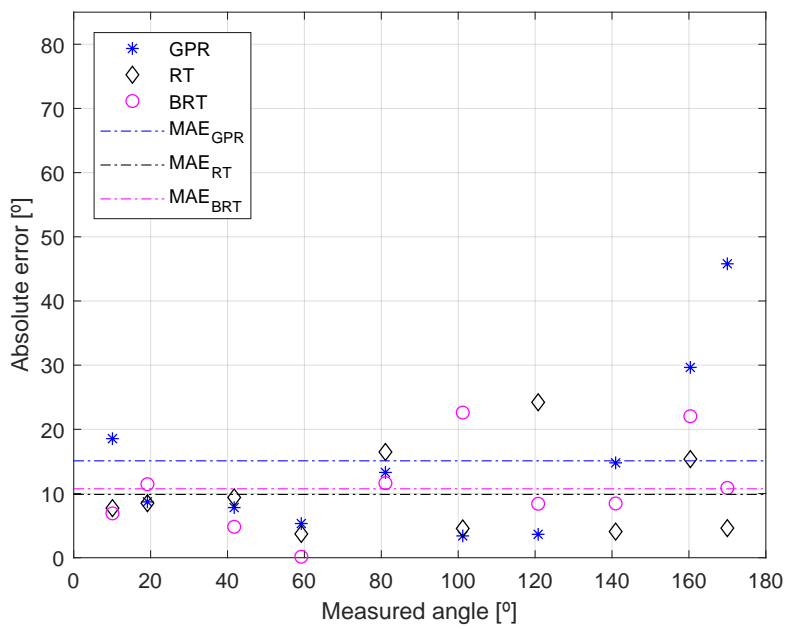

Fig. 8. Regression errors results of non-trained data of an unknown user when models are trained in a dataset obtained from 4 people.

Table 2. Summary of the errors of the regression models in degrees

\begin{tabular}{ccccccc}
\hline & \multicolumn{2}{c}{ Single-known } & \multicolumn{2}{c}{ Multiple-known } & \multicolumn{2}{c}{ Multiple-unknown } \\
\hline Models & ME & MAE & ME & MAE & ME & MAE \\
\hline GPR & 9.24 & 3.77 & 18.89 & 8.68 & 45.79 & 15.09 \\
RT & 12.20 & 4.33 & 74.94 & 17.17 & 24.22 & 9.86 \\
BRT & 10.19 & 5.66 & 32.94 & 13.95 & 22.61 & 10.73 \\
\hline
\end{tabular}




\section{Conclusions}

With this method, new applications of robot-initiated pHRI can be developed without the need of external force/tactile sensors that are expensive or hard to deploy and maintain. These applications may include assistive, rescue or surgical robotics. With this approach, information on the location of the human limbs can be enhanced without the need of additional sensors. In pHRI applications, an accurate location of the human forearm is important not only for a safe human arm manipulation, but also for the placement of biomedical devices such as heart-rate or glucose sensors.

The results demonstrate the good performance of the regression methods used in this application. The errors obtained denote these predictors can be used for pHRI applications. However, in future works, a larger dataset, and a greater number of volunteers may be considered, as well as the use of other prediction methods as deep learning.

Future works after this method may include forearm width estimation for the recognition of people, quality of grasping estimation and skin compliance identification for health evaluation.

Acknowledgment. This work was supported by the the Spanish project DPI2015-65186-R, the European Commission under grant agreement BES-2016078237, the Telerobotics and Interactive Systems Laboratory (TaIS Lab) and the Systems Engineering and Automation Department, University of Málaga, Spain.

\section{References}

1. Armendariz, J., García-Rodríguez, R., Machorro-Fernández, F., Parra-Vega, V.: Manipulation with soft-fingertips for safe phri. In: Proceedings of the seventh annual ACM/IEEE international conference on Human-Robot Interaction, pp. 155156. ACM (2012)

2. Birglen, L.: Enhancing versatility and safety of industrial grippers with adaptive robotic fingers. In: IEEE/RSJ International Conference on Intelligent Robots and Systems (IROS), pp. 2911-2916 (2015)

3. Birglen, L., Gosselin, C.: Optimal design of 2-phalanx underactuated fingers. In: Proceedings of the 2004 International Conference on Intelligent Manipulation and Grasping, pp. 110-116 (2004)

4. Birglen, L., Laliberté, T., Gosselin, C.M.: Underactuated robotic hands. Springer (2008)

5. Bowyer, S.A., y Baena, F.R.: Dissipative control for physical human-robot interaction. IEEE Transactions on Robotics 31(6), 1281-1293 (2015)

6. Breiman, L.: Classification and regression trees. Routledge (2017)

7. Cao, Z., Hidalgo, G., Simon, T., Wei, S.E., Sheikh, Y.: Openpose: realtime multi-person $2 \mathrm{~d}$ pose estimation using part affinity fields. arXiv preprint arXiv:1812.08008 (2018)

8. Chow, K., Kemp, C.C.: Robotic repositioning of human limbs via model predictive control. In: Robot and Human Interactive Communication (RO-MAN), 2016 25th IEEE International Symposium on, pp. 473-480. IEEE (2016) 
9. Erickson, Z., Clever, H.M., Turk, G., Liu, C.K., Kemp, C.C.: Deep haptic model predictive control for robot-assisted dressing. In: IEEE International Conference on Robotics and Automation (ICRA), pp. 1-8 (2018)

10. Frykberg, E.R.: Medical management of disasters and mass casualties from terrorist bombings: how can we cope? Journal of Trauma and Acute Care Surgery 53(2), 201-212 (2002)

11. Gandarias, J.M., Gómez-de Gabriel, J.M., García-Cerezo, A.J.: Enhancing perception with tactile object recognition in adaptive grippers for human-robot interaction. Sensors 18(3), 692 (2018)

12. Gandarias, J.M., García-Cerezo, A.J., Gómez-de Gabriel, J.M.: CNN-based methods for object recognition with high-resolution tactile sensors. IEEE Sensors Journal (2019)

13. King, C., Chen, T.L., Jain, A., Kemp, C.C.: Towards an assistive robot that autonomously performs bed baths for patient hygiene. In: IEEE/RSJ International Conference on Intelligent Robots and Systems (IROS), pp. 319-324 (2010)

14. L. Breiman: Bagging predictors. Machine learning 24(2), 123-140 (1996)

15. Li, Z., Huang, B., Ye, Z., Deng, M., Yang, C.: Physical human-robot interaction of a robotic exoskeleton by admittance control. IEEE Transactions on Industrial Electronics (2018)

16. Ma, R.R., Odhner, L.U., Dollar, A.M.: A modular, open-source 3d printed underactuated hand. In: IEEE International Conference on Robotics and Automation (ICRA), pp. 2737-2743 (2013)

17. Memar, A.H., Mastronarde, N., Esfahani, E.T.: Design of a novel variable stiffness gripper using permanent magnets. In: IEEE International Conference on Robotics and Automation (ICRA), pp. 2818-2823 (2017)

18. Spiers, A.J., Liarokapis, M.V., Calli, B., Dollar, A.M.: Single-grasp object classification and feature extraction with simple robot hands and tactile sensors. IEEE transactions on haptics 9(2), 207-220 (2016)

19. Stilli, A., Cremoni, A., Bianchi, M., Ridolfi, A., Gerii, F., Vannetti, F., Wurdemann, H.A., Allotta, B., Althoefer, K.: Airexglove - a novel pneumatic exoskeleton glove for adaptive hand rehabilitation in post-stroke patients. In: IEEE International Conference on Soft Robotics (RoboSoft), pp. 579-584 (2018)

20. Williams, C.K.: Prediction with gaussian processes: From linear regression to linear prediction and beyond. In: Learning in graphical models, pp. 599-621. Springer (1998)

21. Yang, C., Zeng, C., Liang, P., Li, Z., Li, R., Su, C.Y.: Interface design of a physical human-robot interaction system for human impedance adaptive skill transfer. IEEE Transactions on Automation Science and Engineering 15(1), 329-340 (2018) 